\title{
Characteristics and Outcomes of COVID-19 (SARS-CoV-2) Positive Pregnant Women Admitted to a Dedicated COVID Hospital in Central India: A Single-center Observational Study
}

\author{
Anupama Dave ${ }^{1}$, Sanjay Avashia ${ }^{2}$, Dharmendra Jhavar ${ }^{3}$, Kirti Sinha $^{4}$, Shivam Gupta ${ }^{5}$
}

\begin{abstract}
Aim and objective: To identify characteristics of coronavirus disease-2019 (COVID-19) infection in pregnancy, maternal, and fetal outcomes and study changes in inflammatory markers and chest imaging findings.

Materials and methods: This study is an observational study on pregnant COVID positive women admitted for treatment in the Maharaja Tukojirao Holkar Dedicated COVID Hospital, Indore, Madhya Pradesh, India from April 17, 2020, to April 30, 2021. There were two notable peaks of infection with different clinical characteristics. Group A included data from April 17, 2020, to February 28, 2021, and group B from March 1, 2021, to April 30,2021 . A detailed comparative analysis was done, comparing clinical parameters, investigational findings, and outcomes in both groups.

Results: The incidence of severe acute respiratory syndrome coronavirus 2 (SARS-CoV-2) infection in pregnant women based on admissions in our hospital was 1.26/1,000 maternities. Group A had 94 cases, while group B that included the second wave of infection had 103 cases. The demographic profile of women was comparable in both groups. About $84.04 \%$ of women in group A were asymptomatic. In group B, $27.18 \%$ were asymptomatic. Laboratory parameters were deranged more so in group B. In group A, 41.49\% of women reported in labor and there were no mortalities. In group B, $25.24 \%$ reported in labor, those $<31$ weeks were $60.64 \%$, and there were 12 mortalities. Cesarean section was done in $56.52 \%$ of group A, $39.06 \%$ of group B, and there were $12.5 \%$ of abortions. All of the babies who delivered by vaginal route or cesarean section tested negative within 12 hours of delivery irrespective of maturity.

Conclusion: In our study, we noticed two notable peaks of SARS-CoV-2 infection, first onset was from April 2020 which gradually declined till February 2021 but majority of pregnant women remained asymptomatic, those at term delivered, others recovered within 8 to 14 days, and majority did not require any advanced treatment or oxygen support. Starting from April 2021, there was a resurgence of cases with moderateto-severe disease, morbidities, and even deaths. All novel management options were given on individualized basis including remdesivir, IVIG, and bevacizumab, which proved to be lifesaving in some. All babies delivered tested were SARS-CoV- 2 negative and there was a good neonatal outcome.
\end{abstract}

Keywords: COVID-19, COVID-19 infection in pregnancy, Maternal and neonatal outcome, Maternal outcome, SARS-CoV-2.

Journal of South Asian Federation of Obstetrics and Gynaecology (2021): 10.5005/jp-journals-10006-1919

\section{INTRODUCTION}

After nearly 1 year of attack by the deadly virus, the whole world continues to suffer the disease on and off which is coming up in waves with mutations of the foresaid virus; if we look back to its origin and history, it was in December 2019 that pneumonia of unknown cause was detected in Wuhan, China and reported to the World Health Organization (WHO). ${ }^{1}$ The novel coronavirus, severe acute respiratory syndrome coronavirus 2 (SARS-CoV-2) was found to be the causative agent and the disease subsequently named coronavirus disease-2019 (COVID-19). ${ }^{2}$ The WHO declared the outbreak a pandemic on March 11, 2020.

It initially affected adults and pregnant women who were not spared. Pregnancy as such is an immunocompromised state but whether the normal immunologic changes of pregnancy or the viral structure of SARS-CoV-2 affect the occurrence and course of response to COVID-19 is under constant evaluation. Pregnant women were considered a high-risk group because of concerns about the effect of COVID-19 on them during and after pregnancy, and on their neonates. ${ }^{3}$ Quantification of the rates of COVID-19 infections, its risk factors, clinical manifestations, and outcomes is the key to planning clinical maternal care and management in an evolving pandemic scenario. ${ }^{4}$ As COVID-19 is a novel virus, new evidence is emerging daily and clinical guidelines are vital in providing clinicians with recommendations based on

\footnotetext{
1,4 Department of Obstetrics and Gynecology, MGM Medical College, Indore, Madhya Pradesh, India

${ }^{2}$ Department of Respiratory Medicine, MGM Medical College, Indore, Madhya Pradesh, India

3,5Department of Medicine, MGM Medical College, Indore, Madhya Pradesh, India
}

Corresponding Author: Anupama Dave, Department of Obstetrics and Gynecology, MGM Medical College, Indore, Madhya Pradesh, India, e-mail: anupamadave10@gmail.com

How to cite this article: Dave A, Avashia S, Jhavar D, et al. Characteristics and Outcomes of COVID-19 (SARS-CoV-2) Positive Pregnant Women Admitted to a Dedicated COVID Hospital in Central India: A Single-center Observational Study. J South Asian Feder Obst Gynae 2021;13(4):245-250.

Source of support: Nil

Conflict of interest: None

the latest available evidence. Many societies have developed and published guidance relating to care of pregnant women with COVID-19, but guidance too is rapidly changing and some more studies relating to the clinical characteristics and maternal and fetal outcomes are the need of the hour. 
A dedicated COVID hospital was started at Maharaja Tukojirao Holkar Hospital, Indore, Madhya Pradesh, India, to cater to the increased load of COVID-19 cases in the city. A significant number of COVID-19 positive pregnant women were also admitted to our obstetric wing. In India, vaccination has not been started for pregnant and lactating women till now, which may be one of the reasons why they are still being infected. A detailed analysis was done with the aim that it would help significantly in formulating individual protocols for better management.

\section{Aims and Objectives}

The primary aim of the study was to identify the characteristics of COVID-19 in pregnancy and study the maternal and fetal outcomes in women affected by this infection. In addition, to study the changes in inflammatory markers and chest imaging findings in these women.

\section{Materials and Methods}

It was an observational study on pregnant COVID positive women admitted for treatment in Maharaja Tukojirao Holkar Dedicated COVID Hospital, Indore, Madhya Pradesh, from April 17, 2020, to April 30, 2021.

The records of these patients were examined and analyzed for the course taken by the disease in 197 pregnant women.

Their demographic data, detailed obstetric history, risk factors, and clinical presentation were taken into consideration. All routine and special investigations, sepsis profile along with an X-ray chest, were done. Diagnosis of the presence of COVID-19 infection was done through reverse transcriptase-polymerase chain reaction (RT-PCR)/rapid antigen test (RAT) positive status. Being a limited resource center, facilities of $C T$ were not there, and disease was categorized on the basis of clinical and radiological findings obtained from chest X-rays on admission.

During the course of the study, it was seen that there were two notable peaks of infection with different clinical characteristics in each. So, a detailed comparative analysis was further done, comparing clinical parameters, investigational findings, and outcomes in both groups.

Group A included pregnant women who were admitted from April 17, 2020, to February 28, 2021.

Group B included pregnant women admitted from March 1, 2021, to April 30, 2021.

This kind of distribution was done because it was speculated that the second wave was as a result of a mutant variant of SARSCoV-2 virus speculated as B.1.617, and the pregnant women who were infected were included in group $B$.

Investigations done were $\mathrm{CBC}$, liver function test (LFT), renal function test (RFT), lipid profile, S. electrolytes, D-Dimer, c-reactive protein (CRP), lactate dehydrogenase (LDH), interleukin-6 (IL-6), and procalcitonin (PCT) and electrocardiography (ECG). Random blood sugar monitoring was done by a diabetic team.

The data were collected from observation checklist, questionnaire (semi-structured, pretested), and patients' discharge cards and records.

A departmental protocol was made on the basis of existing international and national guidelines and was followed depending on individual characteristics and requirements (Flowchart 1). An informed consent of all patients at the time of admission was taken after a detailed explanation regarding prognosis and novel methodology of treatment.

The approval was obtained from the institutional ethical committee, and appropriate statistical analysis was done.

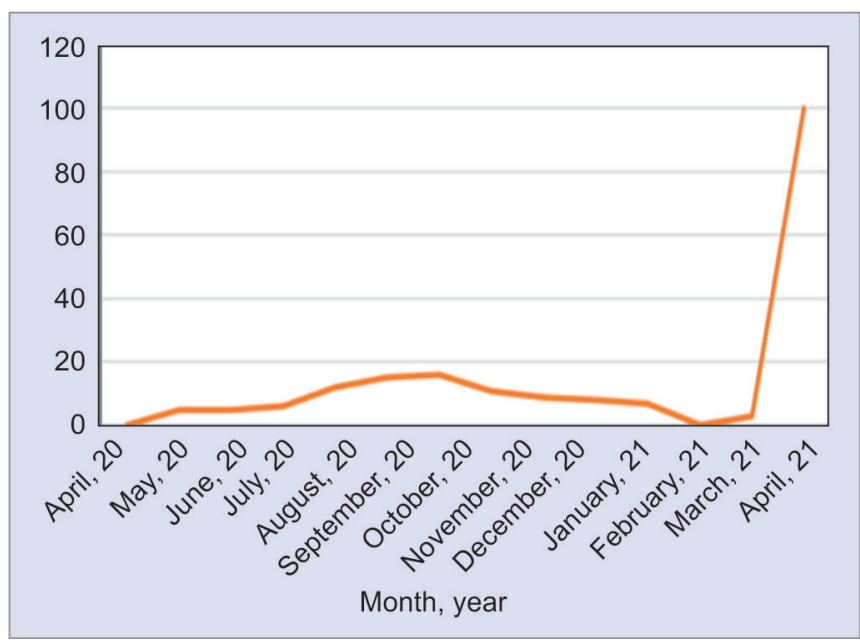

Fig. 1: Distribution of cases month wise

\section{Results}

Maharaja Tukojirao Holkar Hospital is a dedicated COVID hospital attached to the MGM Medical College, Indore, Madhya Pradesh. It is a 400-bedded COVID hospital and has an intensive care unit of 80 beds, which is fully equipped. From its start in April 2020 till April $30,2021,7,044$ patients were admitted, out of which 197 pregnant women were managed in our obstetric wing.

After a detailed analysis of patient characteristics and outcomes of these 197 COVID positive pregnant women, we arrived at the following observations.

The incidence of SARS-CoV-2 infection in pregnant women based on admissions in our hospital was 1.26/1,000 maternities. An analysis of distribution of cases month wise during the study period (Fig. 1) showed the presence of two notable peaks of SARS-CoV-2 infection. Group A had 94 cases while group B that included the second wave of infection had 103 cases. The demographic profile of women was comparable in both groups (Table 1).

In group A, 84.04\% were asymptomatic whereas group B women were reported with symptoms, only $27.18 \%$ were asymptomatic (Table 2). The commonest symptom in group A was fatigue in $8.51 \%$, and in group $B$, respiratory symptoms were commonly seen in $42.72 \%$.

Deranged laboratory parameters were seen more in women of group B (Table 3). In group A, $61.7 \%$ of women had an X-ray COX-RADS score of 0 and none had score of 4 , but in group $B, 29.12 \%$ had a score of $0,20.38 \%$ score $1,12.62 \%$ score $2,20.38 \%$ score 3 , and $17.50 \%$ score 4 (Table 4 ).

In group A, $41.49 \%$ of women reported in labor and there were no mortalities. In group B, $25.24 \%$ reported in labor, majority of women who were admitted were not in labor, $<31$ weeks $60.64 \%$, and there were 12 mortalities (Table 5). Cesarean section in group A was $56.52 \%$ while in group B $39.06 \%$, and there were $12.5 \%$ of abortions (Table 6). All of the babies who were delivered by vaginal route or cesarean section tested negative within 12 hours of delivery irrespective of maturity (Fig. 2).

\section{Discussion}

The effects of COVID-19 on pregnancy are largely unknown; characteristics and facts are constantly changing perhaps due to mutations in the virus itself. Maternal and fetal outcomes in COVID-19 pregnancies can only be defined after a detailed research. 


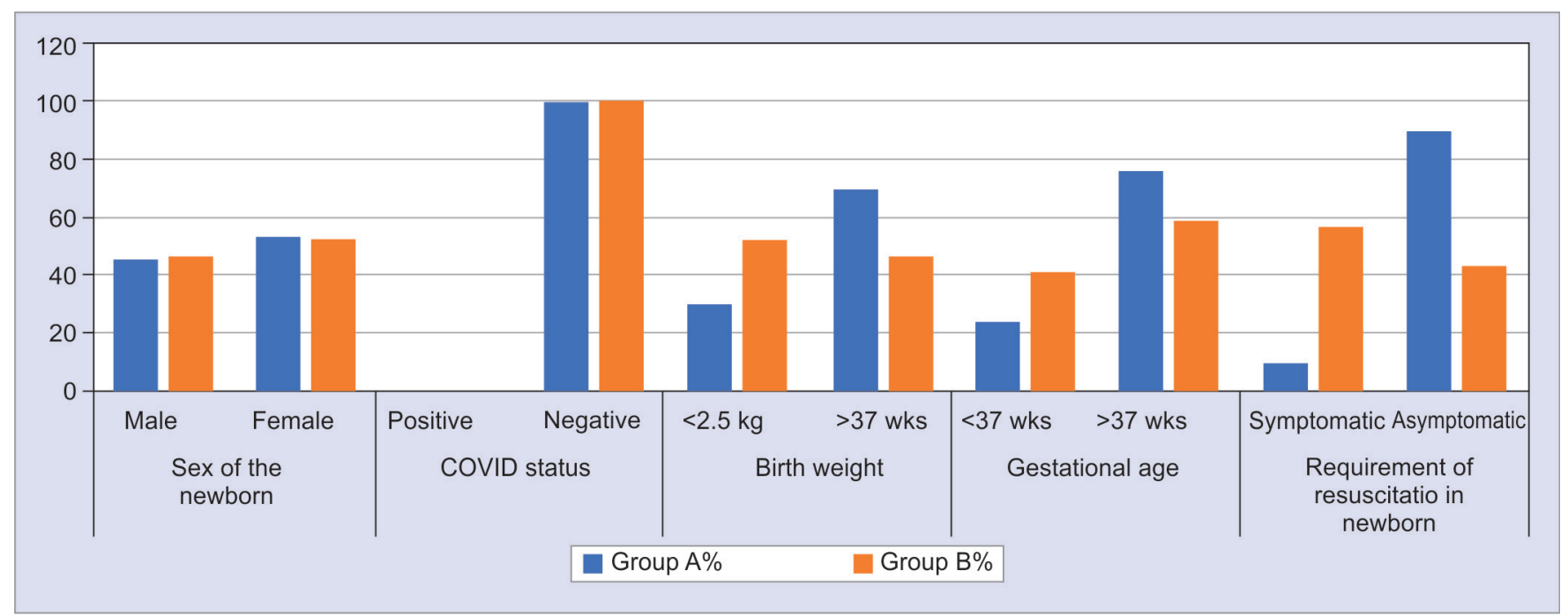

Fig. 2: Neonatal outcomes in COVID-19 positive mothers

Flowchart 1: Departmental protocol

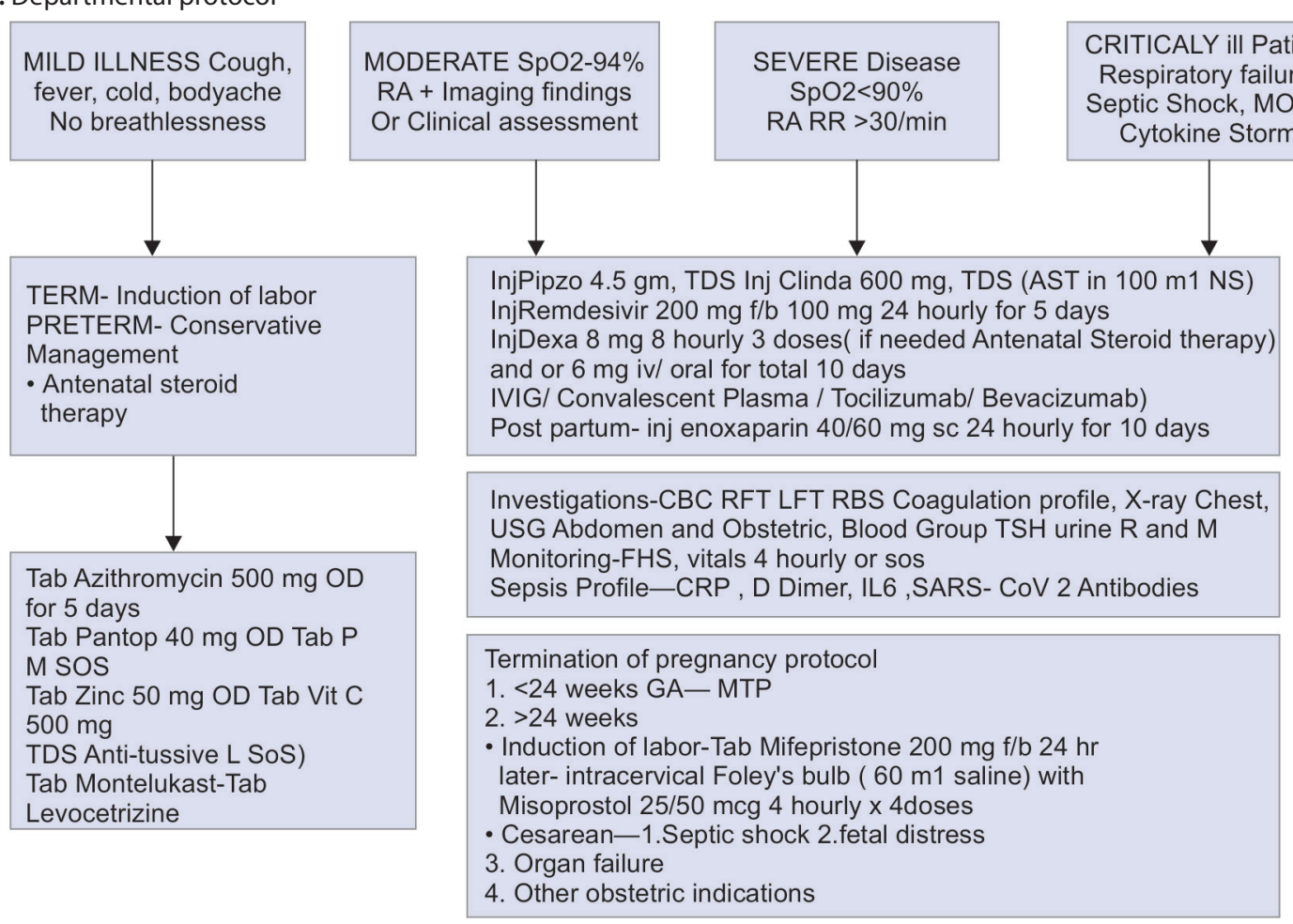

WHO has not found a significant difference in clinical symptoms between nonpregnant and pregnant women of reproductive age. ${ }^{5,6}$ Pregnant women did not appear to be at higher risk of developing more severe disease as compared to the general population as a whole at that particular time frame. During the study period, a total number of 15,639 antenatal cases were admitted to our non-COVID facility, the Department of Obstetrics and Gynecology, Maharaja Yeshwant Rao Holkar Hospital (MYH), whereas in the Maharaja Tukojirao Holkar Dedicated COVID Hospital, it was 197 so an overall incidence of 1.26 per 1,000 maternities. In a report from UK using UKOSS, the estimated incidence in pregnancy was 4.9 (95\% confidence interval 4.5-5.4) per 1,000 maternities. ${ }^{7}$
Disease was recognized on the basis of a galaxy of symptoms, but no single symptom was diagnosed.

In a report from the Center for Disease Control and Prevention (CDC) COVID-19 Response Pregnancy and Infant Linked Outcomes Team by Zambrano et al.:8 on 23,000 pregnant and 386,000 nonpregnant females of reproductive age with symptomatic laboratory-confirmed SARS-CoV-2 infection, the frequency percentage of the most common symptoms in each of the pregnant vs nonpregnant groups reported was cough $-50.3 / 51.3$, headache-42.7/54.9, muscle aches-36.7/45.2, fever-32.0/39.3, sore throat-28.4/34.6, shortness of breath-25.9/24.8, and new loss of taste or smell-21.5/24.8. Other symptoms that occurred 
Table 1: Demographic distribution of COVID-positive women

\begin{tabular}{llcc}
\hline \multirow{2}{*}{ Variable } & & \multicolumn{2}{c}{ Frequency } \\
\cline { 3 - 4 } Age & $18-25$ & Group A (\%) & Group B (\%) \\
& $25-30$ & $37(47.87)$ & $51(49.51)$ \\
Gestational age & $30-35$ & Preterm & $39(37.86)$ \\
(week) & & $43(45.74)$ & $58(56.31)$ \\
& Term & & \\
Parity & Primi & $51(54.25)$ & $45(43.69)$ \\
& Multi & $46(48.94)$ & $36(34.95)$ \\
Socioeconomic & Upper middle class & $5(5.32)$ & $7(6.79)$ \\
status & & & \\
& Lower middle class & $20(21.28)$ & $22(21.36)$ \\
& Upper lower class & $32(34.04)$ & $35(33.98)$ \\
Education & Lower class & $37(39.36)$ & $39(37.86)$ \\
& High school & $7(7.45)$ & $9(8.74)$ \\
& Middle school & $25(26.6)$ & $26(25.24)$ \\
& Primary education/ & $27(28.72)$ & $32(31.07)$ \\
Total & literate & $35(37.23)$ & $36(34.95)$ \\
& Illiterate & 94 & 103 \\
\hline
\end{tabular}

Table 2: Distribution of cases according to symptoms on admission

\begin{tabular}{llcc}
\hline SI. No. & Symptoms & Group A (\%) & Group B (\%) \\
\hline 1 & Respiratory symptoms & $1(1.06)$ & $44(42.72)$ \\
2 & Fever & $2(2.12)$ & $21(20.39)$ \\
3 & SARI & $1(1.06)$ & $18(17.47)$ \\
4 & Diarrhea & $1(1.06)$ & $12(11.65)$ \\
5 & Fatigue & $8(8.51)$ & $18(17.47)$ \\
6 & Asymptomatic & $79(84.04)$ & $28(27.18)$ \\
7 & Anosmia & $2(2.12)$ & $14(13.59)$ \\
\hline
\end{tabular}

SARI, severe acute respiratory infection

Table 3: Distribution according to laboratory parameters in COVID-positive mothers

\begin{tabular}{llcc}
\hline & & \multicolumn{2}{c}{ Number of females with deranged values } \\
\cline { 3 - 4 } SI. No. & Parameters & Group A (\%) & Group B (\%) \\
\hline 1 & CRP & $48(51.06)$ & $65(63.11)$ \\
2 & D-Dimer & $52(55.31)$ & $56(54.37)$ \\
3 & LDH & $17(18.09)$ & $21(20.39)$ \\
4 & PCT (Procalcitonin) & $1(1.06)$ & $9(8.74)$ \\
5 & IL-6 & $11(11.70)$ & $22(21.36)$ \\
& Total & 94 & 103 \\
\hline
\end{tabular}

in $>10 \%$ of each group included nausea, vomiting, fatigue, diarrhea, and rhinorrhea.

In our study during the first wave, majority of women in group A were asymptomatic (84.04\%) and the commonest symptom was fatigue $(8.51 \%)$, while in group $B$, majority presented with respiratory symptoms $(42.72 \%)$ and $27.18 \%$ were asymptomatic. Reason being in the first wave pregnant women were identified because of compulsory screening if a family member turned out to be positive for SARS-CoV-2. Conservative treatment was given to asymptomatic women remote from term $25 / 94$ in group $A$, and they delivered later on after recovery from the infection.

Our findings were similar to a systematic review by Allotey et al. ${ }^{9}$ in which $7 \%$ of pregnant women universally screened for COVID-19 tested positive, $73 \%$ were asymptomatic; they also reported that pregnant women were more likely to be asymptomatic than nonpregnant in the same age-group. In a systematic review by Yanes-Lane et al., ${ }^{10} 95 \%$ (95\% Cl 45-100\%) of COVID-19 infections in pregnant persons were asymptomatic, while 59\% (95\% Cl 49-68\%) remained asymptomatic through follow-up.

After considering symptomatology, specific laboratory parameters were found to be deranged in SARS-CoV-2 infection but at the same time, we must consider that there are changes in laboratory values because of pregnancy. Leukocytosis can be normal in pregnancy, and some of the other laboratory findings overlap with those caused by pregnancy-related disorders (e.g., thrombocytopenia and elevated liver chemistries in preeclampsia with severe features).

In a systematic review by Allotey et al., ${ }^{9}$ altered laboratory parameters were seen which included raised $\mathrm{C}$-reactive protein levels (49\%), lymphopenia (33\%), leukocytosis (26\%), elevated procalcitonin level (23\%), abnormal liver chemistries (15.4\%), and thrombocytopenia (6.6\%).

In our study in the first wave, i.e., group A changes were seen in laboratory parameters but in group $B$ derangements were more pronounced as majority of women were symptomatic.

Radiographic imaging is an important aspect of identifying severity of disease in SARS-CoV-2 infection and needs to be done in pregnant women considering the fact that benefit outweighs the risk and a single $X$-ray chest with shielding, or a CT scan causes only minimal radiation exposure to the fetus, i.e., 0.005 to $0.01 \mathrm{mGy}$ and 0.01 to $0.6 \mathrm{mGy}$, respectively, noted side effects have been seen to occur from high doses $>610 \mathrm{mGy}$ radiation exposures to the fetus. ${ }^{11}$

Chest radiographs may be normal in early or mild disease. In a systematic review by Oshay et al. ${ }^{12}$ in 427 pregnant patients diagnosed with COVID-19, the most frequently encountered pulmonary findings on chest computed tomography were groundglass opacities (77\%), posterior lung involvement (73\%), multilobar involvement (72\%), bilateral lung involvement (69\%), peripheral distribution (68\%), and consolidation (41\%). Our hospital was converted in emergency to a COVID facility so only X-ray facilities

Table 4: Distribution of cases according to X-ray findings (COX-RADS)

\begin{tabular}{lcclcc}
\hline SI. No. & Scoring & Score & Degree of CXR involvement & Group A (\%) & Group B (\%) \\
\hline 1 & 0 & 0 & No chest involvement & $58(61.70)$ & $30(29.12)$ \\
2 & I & $1,2,3,4$ & Mild chest involvement & $31(32.98)$ & $21(20.38)$ \\
3 & II & 5,6 & Moderate chest involvement & $4(4.25)$ & $13(12.62)$ \\
4 & III & $7,8,9$ & Severe chest involvement & $1(1.06)$ & $21(20.38)$ \\
5 & IV & 10 & Severe form & $0(0)$ & $18(17.50)$ \\
& & & Total & 94 & 103 \\
\hline
\end{tabular}


Maternal and Fetal Outcomes of COVID-19 Infection in Pregnancy: An Observational Study

Table 5: Distribution of cases according to gestational age at admission and outcome

\begin{tabular}{|c|c|c|c|c|c|c|c|c|c|}
\hline \multirow[b]{3}{*}{ SI. No. } & \multirow{3}{*}{$\begin{array}{l}\text { Gestational age } \\
\text { at symptom onset }\end{array}$} & \multirow[b]{3}{*}{ No. } & \multicolumn{3}{|c|}{ Group A } & \multirow[b]{3}{*}{ No. } & \multicolumn{3}{|c|}{ Group B } \\
\hline & & & \multicolumn{3}{|c|}{ Outcome } & & \multicolumn{3}{|c|}{ Outcome } \\
\hline & & & Conservative & Termination & Mortality & & Conservative & Termination & Mortality \\
\hline 1 & $<22$ weeks & $8(8.51 \%)$ & 7 & 1 & 0 & $11(10.67 \%)$ & 9 & 2 & 0 \\
\hline 2 & $22-27$ weeks & 3 (3.19\%) & 3 & 0 & 0 & 9 (8.74\%) & 6 & 3 & 2 \\
\hline 3 & 28-31 weeks & $3(3.19 \%)$ & 3 & 0 & 0 & $16(15.53 \%)$ & 8 & 8 & 1 \\
\hline 4 & 32-36 weeks & $14(14.89 \%)$ & 5 & V-05, L-04 & 0 & $22(21.34 \%)$ & 8 & V-06, L-08 & 2 \\
\hline 5 & $>37$ weeks & 24 (25.53\%) & 4 & V-06, L-14 & 0 & 16 (15.53\%) & 5 & V-06, L-05 & 4 \\
\hline 6 & Peripartum & 39 (41.49\%) & 0 & V-18, L-21 & 0 & 26 (25.24\%) & 0 & V-12, L-14 & 2 \\
\hline \multirow[t]{2}{*}{7} & Postpartum & $3(3.19 \%)$ & 3 & 0 & 0 & $3(2.91 \%)$ & 3 & 0 & 1 \\
\hline & Total & 94 & 25 & 69 & 0 & 103 & 39 & 64 & 12 \\
\hline
\end{tabular}

$V$, stands for vaginal delivery; $L$, stands for lower segment cesarean section

Table 6: Distribution of cases according to mode of delivery

\begin{tabular}{llccc}
\hline Outcome & & Group A (\%) & Group B (\%) & Total \\
\hline Abortion & Induced & $00(0.00)$ & $8(12.5)$ & 8 \\
& Spontaneous & $1(\mathrm{~L} 45)$ & $0(0.00)$ & 1 \\
$\begin{array}{l}\text { Term vaginal } \\
\text { deliveries }\end{array}$ & Induced & $1(1.45)$ & $3(4.69)$ & 4 \\
& Spontaneous & $24(34.78)$ & $12(18.75)$ & 36 \\
$\begin{array}{l}\text { Preterm } \\
\text { vaginal } \\
\text { deliveries }\end{array}$ & Induced & $1(1.45)$ & $8(12.5)$ & 9 \\
$\begin{array}{l}\text { Cesarean } \\
\text { sections }\end{array}$ & Preterm & $7(10.14)$ & $13(20.31)$ & 20 \\
& Term & $32(46.38)$ & $12(18.75)$ & 55 \\
Total & & 69 & 64 & 133 \\
\hline
\end{tabular}

were there. Plain chest radiograph (CXR), although less sensitive than chest $C T$, is usually the first-line imaging modality used for patients with symptomatic SARS-CoV-2 infection. The relation between radiological changes in CXR and clinical severity of the disease in symptomatic patients with COVID-19 has not been fully studied, but we tried COX-RADS a proposed scoring by De Sanctis et al. ${ }^{13}$ There is no such study on pregnant COVID positive women, and our motive was only to identify the severity of lung involvement, so that individualized treatment may be started.

It was seen that majority of women in group A (61.70\%) had normal X-ray findings and a score of 0 as they were asymptomatic. In group $B$ as the severity of COVID-19 infection was more during this period, only $29.2 \%$ had a score of 0 while majority $(50.50 \%)$ women had moderate-to-severe disease.

Gestational age at the time of COVID-19 infection is an important factor in deciding further management. In our management protocol (Flowchart 1), individualized decision-making regarding continuation of pregnancy was the key to successful management. It was made on the basis of severity of infection categorization depending on clinical and imaging findings. In group A, 41.49\% of women were reported in labor of which 18/39 delivered vaginally and cesarean section was done in $21 / 39$, almost all were asymptomatic. Those $<31$ weeks were managed conservatively. But in group $B$ as majority had mild-to-severe disease, the decision of termination was taken in view of maternal benefit in 64/103 cases and conservative management was done in 39/103 cases.
A systematic review by Allotey et al. ${ }^{9}$ including over 11,000 pregnant and recently pregnant women with suspected or confirmed COVID-19 reported that $17 \%$ delivered before 37 weeks and $65 \%$ delivered by cesarean. Most preterm deliveries were iatrogenic; only $6 \%$ were spontaneous. In our study, cesarean section was done in $56.52 \%$ in group $A$ in which $10.14 \%$ were preterm, while in group B $39.06 \%$ in which $20.31 \%$ were preterm. Moreover, 2.9 and $12.5 \%$ of preterm vaginal deliveries were spontaneous in groups $A$ and $B$, respectively. Due to severe infection and lung involvement, induction was done in 1.45 and $12.5 \%$ of both groups, respectively. During the second wave due to the same reason, there were $12.5 \%$ induced abortions in group B.

In viral infections, transmission to the neonate is a major concern during pregnancy as well as during and after birth. With the novel virus, there were many varied reports.

In a systematic review by Kotlyar et al. ${ }^{14}$ of infants born to 936 COVID-19 infected mothers, neonatal viral RNA testing was positive in $27 / 936$ (2.9\%) nasopharyngeal samples taken immediately after birth or within 48 hours of birth, $1 / 34$ cord blood samples, and 2/26 placental samples; in addition, $3 / 82$ neonatal serologies were immunoglobulin M (IgM) positive for SARS-CoV-2. In addition, in the CDC COVID-19 Response Pregnancy and Infant Linked Outcomes Team report by Woodworth et al. ${ }^{15}$ of over 5,000 pregnant persons with laboratory-confirmed SARS-CoV-2 infection, $2.6 \%$ of the 610 infants with available SARS-CoV-2 test results had a positive test, primarily those born to persons with infection at delivery.

In utero transmission typically occurs via a hematogenous route but sometimes via the ascending route. Viremia rates in patients with COVID-19 may be low (1\%) in one study by Penfield et al., ${ }^{16}$ but also found to be higher in severe disease and transient in a study by Patane et al., ${ }^{17}$ suggesting placental seeding and in utero transmission may not be common.

In studies conducted, most of the placentas studied had no evidence of infection, but the virus had been identified in few cases; there are reports of positive vaginal swabs and positive amniotic fluid, ${ }^{18-22}$ which may suggest the ascending route of infection and intrapartum transmission through vaginal secretions. Viral shedding in maternal feces has been reported, so fecal contamination of the perineum could theoretically be a source of intrapartum transmission, although there is no evidence of protective effect of cesarean. Postnatal transmission can occur through ingestion of breast milk or from an infected mother (or other caregivers) to her infant through respiratory or other infectious secretions.

In our study, however, all neonates were tested within 12 hours of birth by RT-PCR as negative; no evidence of infection was noted 
in them on follow-up also. In contrary to this, in a study from South India by Nambiar et al., ${ }^{23} 21.42 \%$ of babies who delivered vaginally tested positive, while $10.83 \%$ of babies delivered by cesarean section were positive.

\section{Conclusion}

In our study, we noticed two notable peaks of SARS-CoV-2 infection, first onset was from April 2020 which gradually declined till February 2021, but majority of pregnant women who tested positive remained asymptomatic and those who were term delivered, others recovered within 8 to 14 days during that period majority did not require any advanced treatment or oxygen support.

But starting from April 2021, there was a resurgence of cases in whom moderate-to-severe disease was noted, it was associated with morbidities and even deaths. All novel management options were given on individualized basis including remdesivir, IVIG, and bevacizumab, which proved to be lifesaving in some.

One fact common to both groups was that the babies who were delivered were tested SARS-CoV-2 negative and there was a good neonatal outcome; they were either breastfed or given expressed breast milk and all precautions were adhered to strictly.

\section{Clinical Significance}

Our study emphasizes the importance of screening pregnant women at point of contact specially those at the time of admission to the hospital in labor or for any other reason. Those tested positive for SARS-CoV-2 must be further investigated and the importance of early admission in a COVID hospital if any symptoms arise must not be overlooked. Early admission in case of lung involvement and delivery is a lifesaving option. However, more research and studies on a large population are required to formulate guidelines for more efficient and evidence-based management.

\section{References}

1. World Health Organization. Rolling updates on coronavirus disease (COVID-19). 2020. Available from: https://www.who.int/emergencies/ diseases/novel-coronavirus-2019/events-as-they-happen.

2. World Health Organization. Naming the coronavirus disease (COVID-19) and the virus that causes it. 2020. Available from: https:// www.who.int/emergencies/diseases/novel-coronavirus-2019/ technical-guidance/naming-the-coronavirus-disease-(covid-2019)and-the-virus-that-causes-it.

3. Cabinet Office. Guidance. Staying alert and safe (social distancing). Coronavirus (COVID-19) guidance and support. 2020. Available from: https://www.gov.uk/government/publications/staying-alert-andsafe-social-distancing/staying-alert-and-safe-social-distancing [Accessed May 24, 2020].

4. RCOG. (COVID-19) infection in pregnancy. Available from: https:// www.rcog.org.uk/en/guidelines-research-services/guidelines/ coronavirus-pregnancy/.

5. Chen $\mathrm{H}, \mathrm{Guo} J$, Wang $\mathrm{C}$, et al. Clinical characteristics and intrauterine vertical transmission potential of COVID-19 infection in nine pregnant women: a retrospective review of medical records. Lancet 2020;395(10226):809-815. DOI: 10.1016/S0140-6736(20)30360-3.

6. Aylward B, Liang W, Dong X, et al. Report of the WHO China joint mission on coronavirus disease2019 (COVID-19) [Internet]. Beijing: World Health Organisation; 2020.

7. Knight $\mathrm{M}$, Bunch $\mathrm{K}$, Vousden $\mathrm{N}$, et al. Characteristics and outcomes of pregnant women admitted to hospital with confirmed SARSCoV-2 infection in UK: national population based cohort study. BMJ2020;369:m2107 Available from: https://doi.org/10.1136/bmj.m2107.
8. Zambrano LD, Ellington $S$, Strid $P$, et al. Update: characteristics of symptomatic women of reproductive age with laboratory-confirmed SARS-CoV-2 infection by pregnancy status - United States, January 22-October 3, 2020. MMWR Morb Mortal Wkly Rep 2020;69(44):1641. DOI: $10.15585 / \mathrm{mmwr} . \mathrm{mm} 6944 \mathrm{e}$.

9. Allotey J, Stallings $\mathrm{E}$, Bonet $\mathrm{M}$, et al. Clinical manifestations, risk factors, and maternal and perinatal outcomes of coronavirus disease 2019 in pregnancy: living systematic review and meta-analysis. BMJ 2020;370:m3320. DOI: 10.1136/bmj.m3320.

10. Yanes-Lane M, Winters N, Fregonese F, et al. Proportion of asymptomatic infection among COVID-19 positive persons and their transmission potential: a systematic review and meta-analysis. PLoS One 2020;15(11):e0241536. DOI: 10.1371/journal.pone.0241536.

11. Tremblay $E$, Thérasse $E$, Thomassin-Naggara I, et al. Quality initiatives: guidelines for use of medical imaging during pregnancy and lactation. Radiographics 2012;32(3):897. DOI: 10.1148/rg.323115120.

12. Oshay RR, Chen MYC, Fields BKK, et al. COVID-19 in pregnancy: a systematic review of chest CT findings and associated clinical features in 427 patients. Clin Imaging 2021;75:75. DOI: 10.1016/j. clinimag.2021.01.004.

13. De Sanctis V, Bedair EMA, Soliman AT, et al. Proposed scoring system for evaluating clinico-radiological severity of COVID-19 using plain chest X-ray (CXR) changes (CO X-RADS): preliminary results. Acta Biomed 2020;91(4):e2020172. DOI: 10.23750/abm.v91i4.10664.PMID: 33525220; PMCID: PMC7927462.

14. Kotlyar AM, Grechukhina O, Chen A, et al. Vertical transmission of coronavirus disease 2019: a systematic review and metaanalysis. Am J Obstet Gynecol 2021;224(1):35. DOI: 10.1016/j. ajog.2020.07.049.

15. Woodworth KR, Olsen EO, Neelam V, et al. Birth and infant outcomes following laboratory-confirmed SARS-CoV-2 infection in pregnancy - SET-NET, 16 Jurisdictions, March 29-October 14, 2020. MMWR Morb Mortal Wkly Rep 2020;69(44):1635. DOI: 10.15585/mmwr. mm6944e2.

16. Penfield CA, Brubaker SG, Limaye MA, et al. Detection of severe acute respiratory syndrome coronavirus 2 in placental and fetal membrane samples. Am J Obstet Gynecol MFM 2020;2(3):100133. DOI: 10.1016/j. ajogmf.2020.100133.

17. Patanè L, Morotti D, Giunta MR, et al. Vertical transmission of coronavirus disease 2019: severe acute respiratory syndrome coronavirus 2 RNA on the fetal side of the placenta in pregnancies with coronavirus disease 2019-positive mothers and neonates at birth. Am J Obstet Gynecol MFM 2020;2(3):100145. DOI: 10.1016/j.ajogmf.2020.100145.

18. Huntley BJF, Huntley ES, Di Mascio D, et al. Rates of maternal and perinatal mortality and vertical transmission in pregnancies complicated by severe acute respiratory syndrome coronavirus 2 (SARS-Co-V-2) infection: a systematic review. Obstet Gynecol 2020;136(2):303. DOI: 10.1097/AOG.0000000000004010.

19. Qiu L, Liu X, Xiao M, et al. SARS-CoV-2 is not detectable in the vaginal fluid of women with severe COVID-19 infection. Clin Infect Dis 2020;71(15):813. DOI: 10.1093/cid/ciaa375.

20. Pique-Regi R, Romero R, Tarca AL, et al. Does the human placenta express the canonical cell entry mediators for SARS-CoV-2? Elife 2020;9:e58716. DOI: 10.7554/eLife.58716.

21. Hecht JL, Quade B, Deshpande V, et al. SARS-CoV-2 can infect the placenta and is not associated with specific placental histopathology: a series of 19 placentas from COVID-19-positive mothers. Modern Pathology. 2020 Nov;33(11):2092-2103. Available from: https://doi. org/10.1038/s41379-020-0639-4.

22. Rasmussen $S A$, Lyerly $A D$, Jamieson DJ. Delaying pregnancy during a public health crisis - examining public health recommendations for Covid-19 and beyond. N Engl J Med 2020;383(22):2097-2099. DOI: 10.1056/NEJMp2027940.

23. Nambiar SS, Ajith S, Reshmi VP. Assessing disease outcome in COVID-19 pregnancies in a tertiary referral center in South India: a single-center retrospective cohort study. J South Asian Feder Obst Gynae 2020;12(5):335-339. DOI: 10.5005/jp-journals-10006-1822. 